\title{
A Mesh Sensor Network based on Bluetooth: Comparing Topologies to Crop Monitoring.
}

\author{
Luan H. S. Alves*, Elida Antunes*, Ricardo Ferreira ${ }^{\dagger}$ and José A. M. Nacif* \\ E-mail: \{luan.h.alves, elida.antunes, ricardo, jnacif\}@ufv.br \\ *Instituto de Ciências Exatas e Tecnológicas \\ Universidade Federal de Viçosa, Florestal, MG, Brazil \\ ${ }^{\dagger}$ Departamento de Informática \\ Universidade Federal de Viçosa, Vicosa, MG, Brazil
}

\begin{abstract}
The growth of the world population increases the demand for food and other products from agriculture. Therefore, it is increasingly needed to enhance and optimize the productivity of farms. Through using a Wireless Sensor Network (WSN) and the application of the internet of things (IoT) technologies, we can better monitor the condition of farm crops. In this work, we designed a Bluetooth Low Energy mesh network joined fluorescence sensors for agricultural crop monitoring, and also we evaluated the suitability of this solution in a crop field scenario.
\end{abstract}

\section{INTRODUCTION}

The current world population of 7.6 billion, is expected to overcome 8.6 billion people in 2030, according to the United Nations (UN) [1], and this population growth raises the demand for food and other products from agriculture. Moreover, agriculture is an important industry that drives billions of dollars every year [2], [3]. For these reasons, there is a growing need for maximizing agricultural production efficiency and optimize the use of production resources.

Wireless Sensors Network (WSN) are widely used and play a vital role in precision agriculture [4] to monitor the conditions of the farm. Farmers use WSNs to reduce uncertainties in decision-making for a better understanding of the information acquired in the crop environment, and thus, to optimize the use of resources (water, pesticides, fertilizers), increase the production, and reduce losses. Recent advances in electronics systems of the Internet of Things (IoT) technologies have allowed the development of small and lowcost sensors capable of communicating through the wireless network. However, the wireless sensors should be low power consumption and have the capacity to transmit over long distances to be feasible in the agricultural environment.

The Bluetooth Low Energy (BLE) has some essential features for precision agriculture: low energy consumption and medium transmission distance. When used in the mesh topology, BLE expands the communication coverage range and scalability. These characteristics support the use of the BLE in a Wireless Sensor Network (WSN) in scenarios where the direct communication between two end-points may not be possible, such as farm scenario.

In this paper, we have built an efficient and automated system for monitoring crops using the BLE mesh network to send measurements of a chlorophyll fluorescence sensor to assist producers in decision-making. The measurement of chlorophyll fluorescence $(\mathrm{CF})$ is a non-invasive and well-known technique for measure plant health, and the use of fluorescence sensors can be an effective method for monitoring crops. In this work, we focus on the sensor communication topology, range, and power consumption.

We organize the paper as follows. Section II studies previous work related to our solution. In Section III we discuss the design of the sensor node as well as the network communication. Section IV describes the sensor network application scenario. In Section V, we examine our results and analyze the suitability of our mesh sensor network in the proposed scenario. Finally, Section VI concludes this paper. 


\section{RELATED WORK}

Applications of the Internet of Things in the agricultural sector has been developed in recent years. However, few works utilize a fluorescence sensor network for crop monitoring. Therefore, in this Section, we present some studies on the use of Bluetooth and its suitability in the farming scenario, and also we present some basics on fluorescence sensors.

C. Gull et al. [6] present in their work a low-cost $\mathrm{CF}$ sensor to monitor plant health. They propose a solution that uses off-the-shelf components in an affordable package, which can measure CF. The solution shows potential as a low-cost fluorometer and opens up the possibility of utilizing multiple sensors in a WSN.

In [7], T. Rault et al. provide a survey of energy efficiency in the wireless sensor network in several categories of applications. This work presents the trade-offs between application requirements and sensor node lifetime. They expose that the main requirements of environmental and agricultural applications are scalability, coverage, and lifetime prolongation, which are met by the BLE mesh network. They also made a comparison with some wireless standards, and the Bluetooth Low Energy and ZigBee are the lowest power consumption wireless standards, although, BLE has higher data rate transmission.

In the same direction of [7], Mekala and P. Viswanathan [8] and Shi et al. [9] study several network communication technologies for agriculture. The Bluetooth Low Energy has been considered in their work, although they do not discuss mesh network over BLE.

P. Zenker et al. [10], examine the applicability of Bluetooth Low Energy for mesh-enabled applications. They used modules equipped with CSR's Bluetooth chip CSR1010 and CSRmesh protocol to study the suitability of Bluetooth Mesh in largescale networks. They conclude that the Bluetooth Mesh is a promising and useful technology for mesh applications. This work also includes a simulation of a network ranging from 100 to 500 nodes placed following a uniform grid. The authors show that the network size does not have a significant impact on end-to-end packet delivery when the nodes are uniformly spatial distributed.
Table I shows a comparison between some wireless standards regarding transmission range, data rate, power consumption, and cost. Our choice for Bluetooth low energy (BLE) is due to its low power consumption and low cost. Although the Bluetooth does not have a wide transmission range, such as the other standards, we can mitigate this issue by using the mesh topology.

TABLE I: Comparison of wireless technologies.

\begin{tabular}{lcccc}
\hline $\begin{array}{c}\text { Wireless } \\
\text { technology }\end{array}$ & Range & $\begin{array}{c}\text { Data } \\
\text { rate }\end{array}$ & $\begin{array}{c}\text { Power } \\
\text { consumption }\end{array}$ & Cost \\
\hline Wi-Fi & $10-100 \mathrm{~m}$ & $6.75 \mathrm{~Gb} / \mathrm{s}$ & High & Low \\
Zigbee & $10-100 \mathrm{~m}$ & $250 \mathrm{~Kb} / \mathrm{s}$ & Low & Medium \\
Lora & $+10 \mathrm{~km}$ & $50 \mathrm{~Kb} / \mathrm{s}$ & Low & Medium \\
Bluetooth & $10 \mathrm{~m}$ & $3 \mathrm{Mb} / \mathrm{s}$ & Medium & Low \\
BLE & $10-50 \mathrm{~m}$ & $1 \mathrm{Mb} / \mathrm{s}$ & Low & Low \\
\hline
\end{tabular}

\section{System ARChitecture}

In this section, we discuss the composition of the sensor node as well as the characteristics of the Bluetooth mesh network topology and the cloud platform. We present the architecture of our system in Fig. 1.

\section{A. Sensor Node}

The measurement of chlorophyll fluorescence is a well-known technique affording robust monitoring [11], and with no harmful consequences for the plant. Therefore, we use the fluorescence sensor illustrated in Fig. 2, capable of distinguishing a healthy plant from an unhealthy plant by measuring the fluorescence emitted by the leaf in the process of photosynthesis. We describe the operation of the sensor as follows: (1) Sensor emits light; (2) The leaf emit the fluorescence; (3) The optical sensor captures this fluorescence; (4) The measured parameters are transmited to the microcontroller; (5) The measured parameters are sent to the node sink through the BLE module. The following components compose each sensor node:

1) LEDs: To stimulate the process of photosynthesis, we use UV and IR LEDs in the 470 $\mathrm{nm}$ and $627 \mathrm{~nm}$ spectrum, respectively.

2) Optical Sensor: The optical sensor we use is the OPT101 monolithic photodiode from Texas Instruments. 


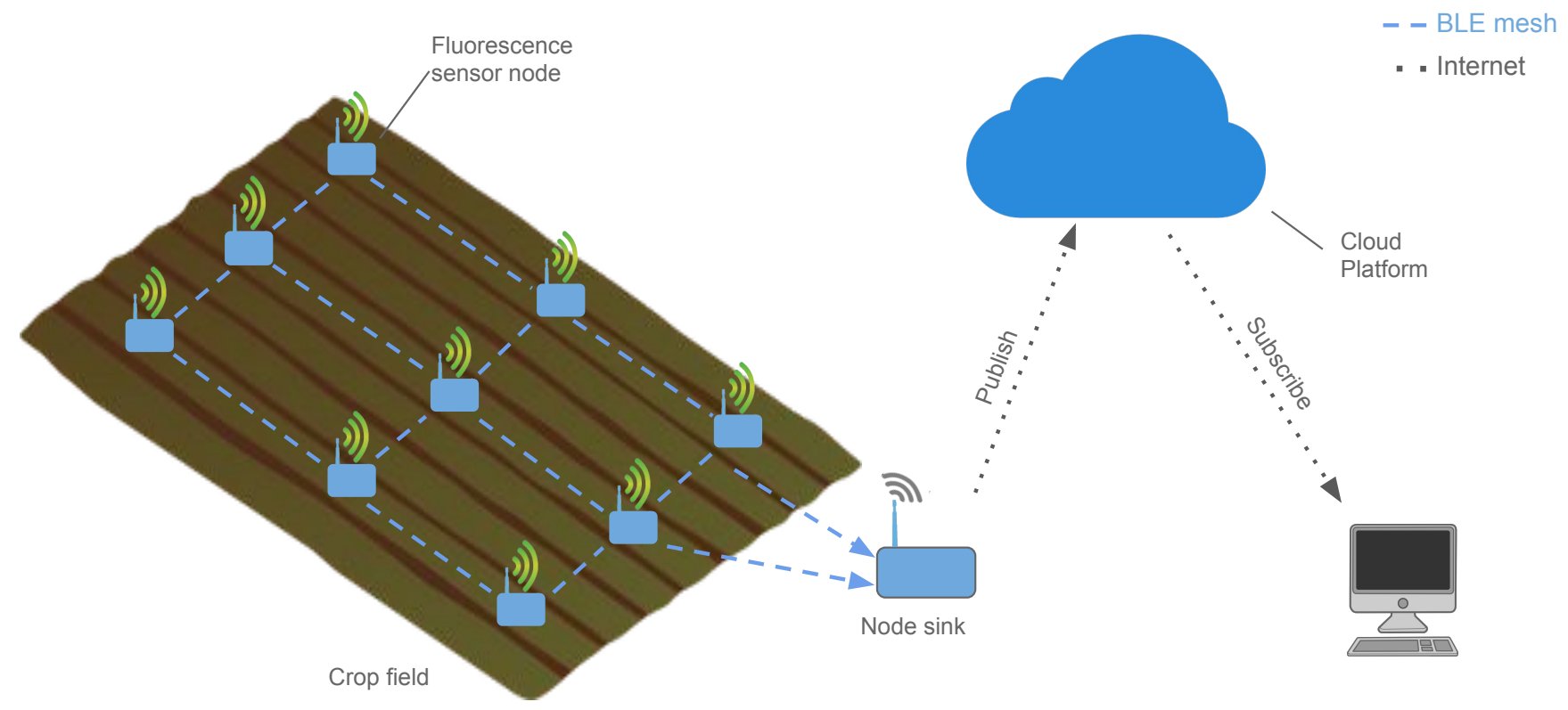

Fig. 1: Architecture of the Wireless Sensor Network monitoring System.

3) Filter: Our interest is that the optical sensor measure fluorescence emitted by the leaf, for this reason, we use a filter to allow the passage of wavelengths between $690 \mathrm{~nm}$ to $740 \mathrm{~nm}$, where is the fluorescence emission peak, and block undesired light out of this spectrum range.

4) Microcontroller: Every sensor node has a microcontroller MSP430G2553 to control the activation of the LEDs and the optical sensor for each measurement cycle.

5) BLE Module: The CSR1010 BLE module [12] with proprietary BLE-based CSRmesh protocol is responsible for sending the data into the mesh network to the node sink.

\section{B. Mesh Communication}

Bluetooth Low Energy (BLE) [13] has been developed explicitly for devices with low battery capacity, low-cost and short and medium range requirements. In terms of power consumption efficiency, BLE is designed so that devices can have a prolonged lifetime.

The Bluetooth uses piconet, Fig. 6a, as standard topology. In this type of network, the slave nodes are only allowed to connect to the master node. It is also possible to use the scatternet, Fig. 6b, topology, which is a chain of piconets connected by a bridge node.

Due to the low power consumption and the restrictions of the standard topology, we configure the sensors in a mesh network over Bluetooth Low Energy. In a mesh network, Fig. 3c, every node can reach any other node of the network, because the nodes transmit their packets and also relay packets received from other nodes. The advantage of this type of topology is that it is easy to expand the range by simply inserting a new node. These characteristics of mesh topology are suitable for our application scenario because we can transfer data over long distances with a low power consumption radio.

CSRmesh [14] developed by Cambridge Silicon Radio (CSR), is a proprietary protocol that operates over Bluetooth low energy. It uses a flooding mechanism to transmit messages across the network, and the advantage of this approach is simplicity, as it does not require the establishment of connections within adjacent BLE devices neither a routing protocol. However, the limitation of this mechanism is the occurrence of bottlenecks in high data traffic condition, or also in the distribution of a random node in a dense network [10]. 


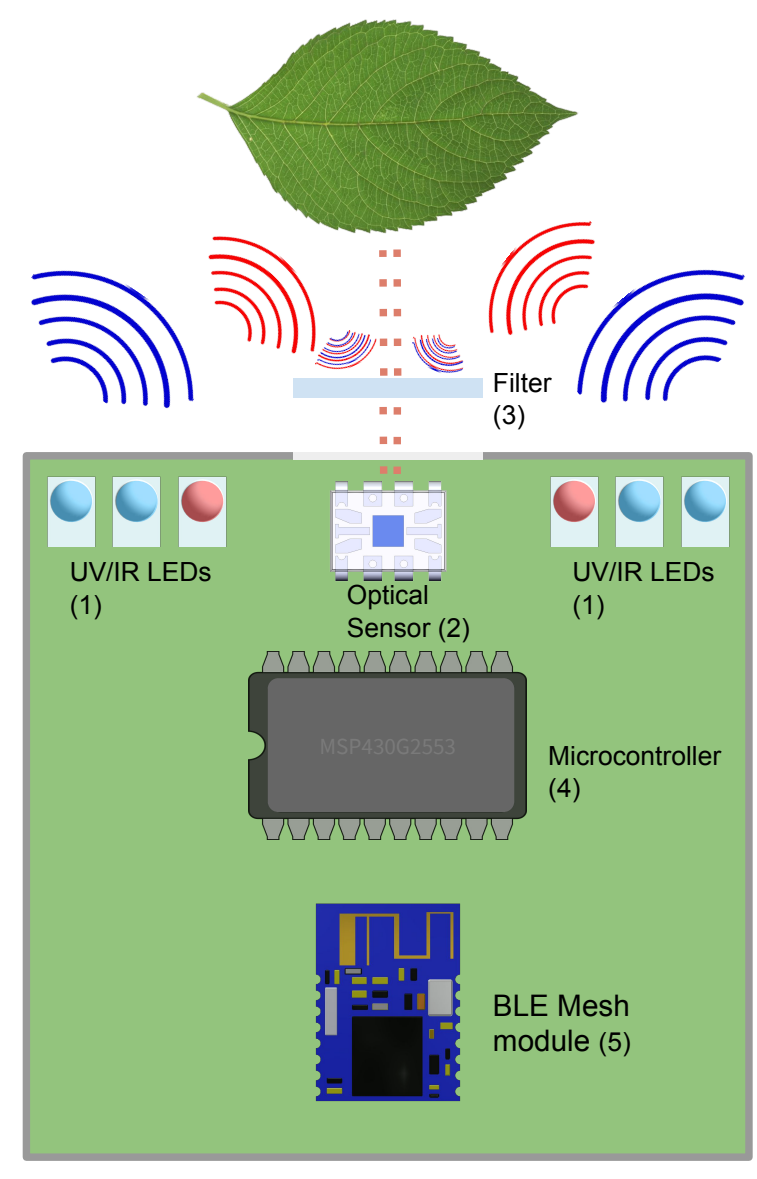

Fig. 2: Overview diagram of the sensor node.

An advantage of Bluetooth, in contrast with other wireless patterns, is its easy integration with other systems. For example, it is possible to place IoT systems in the crop to manage irrigation, humidity, and temperature and connect all via Bluetooth and control and monitoring them by applications embedded in mobile devices such as tablets and smartphones.

\section{Cloud Platform}

After sensor measurements, the node sink receives the data and sends it to the Cloud IoT Core of the Google Cloud Platform, via MQTT, a lightweight machine-to-machine publish/subscribe messaging protocol [15], [16]. The Cloud IoT Core is a service from Google Cloud Platform, that allows easy and secure management of IoT devices, and provide solutions for processing and visualization of the measured data in real-time.

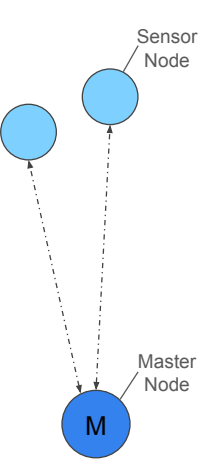

(a) Piconet

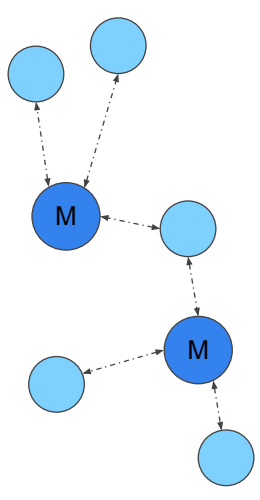

(b) Scatternet

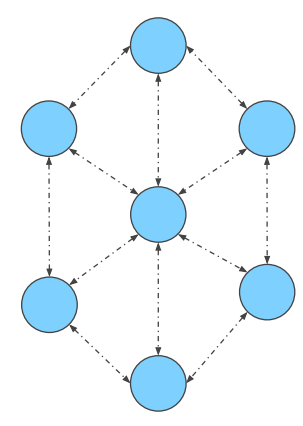

(c) Mesh
Fig. 3: Bluetooth Low Energy topologies.

\section{ApPlicATION SCENARIO}

The application scenario in which we evaluate the suitability of our sensor mesh network is a field crop of 1 hectare (100 x 100 meters). We place nine sensor nodes in a uniform grid pattern separated by 30 meters, such as crop in Fig. 1.

We have made three different tests using the wireless sensor network, considering our scenario. These tests aim to examine the power consumption, communication distance range and also compare the Bluetooth topologies and the minimum number of sensor nodes to have good monitoring coverage.

\section{RESUlts}

In this section, we analyze the data obtained by experiments in the application scenario discussed in Section V and also, we compare between the Bluetooth topologies.

\section{A. Communication Distance}

In this experiment, our focus is on the maximum distance we reach between two nodes. For this reason, there is no distinction between topologies. Table II presents the results obtained in the tests.

\section{TABLE II: Distance Test Results}

\begin{tabular}{lc}
\hline Communication Distance & Range \\
\hline Maximum & $45-50$ meters \\
Safe & $25-30$ meters \\
\hline
\end{tabular}

In our tests, we found that the maximum communication range between nodes is around 45 to 


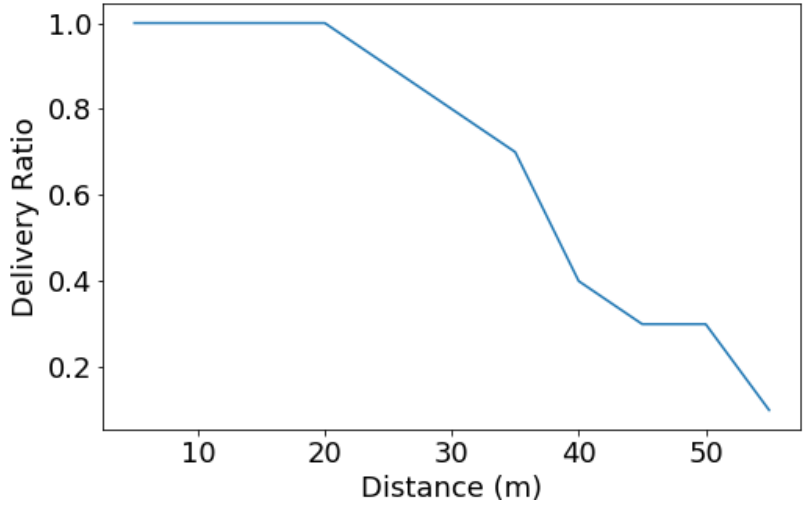

Fig. 4: Maximum distance between modules.

50 meters. However, we noticed that in this range, there were eventual losses in the packets delivery as shown in Fig 4. For this reason, we established the safe distance range of communication, where the occurrence of failures is unlikely. For this reason, it is possible to use Bluetooth in a shortmedium range.

\section{B. Power Consumption}

In this experiment, we have used a power monitor to measure the energy consumption of the BLE module with mesh and without mesh protocol. We have collected 2,000 samples at a rate of $1 \mathrm{kHz}$, and then we calculate the power consumption average. Table III shows the tests results.

TABLE III: Power consumption.

\begin{tabular}{lcc}
\hline Protocol & Idle & Transmitting \\
\hline Mesh & $29.47 \pm 2.72 \mathrm{~mA}$ & $40.55 \pm 2.90 \mathrm{~mA}$ \\
No mesh & $19.56 \pm 1.24 \mathrm{~mA}$ & $22.20 \pm 1.49 \mathrm{~mA}$ \\
\hline
\end{tabular}

In our tests, we verified that the Bluetooth mesh consumes $50.63 \%$ more in idle mode and up to $82,64 \%$ more in transmission mode when compared to regular Bluetooth network. Because of the BLE mesh relay messages from other modules and also its messages.

Further, we estimate the BLE module battery lifetime. Regularly, battery lifetime (BL), Equation 1 , is calculated based on the current consumption rating $(\mathrm{CR})$ in Milliampere $(\mathrm{mA})$ and the capacity of the battery (BC) in Milliampere Hours (mAh). In our results, we include the discharge safe (DS), the percentage of the battery capacity that is never used.

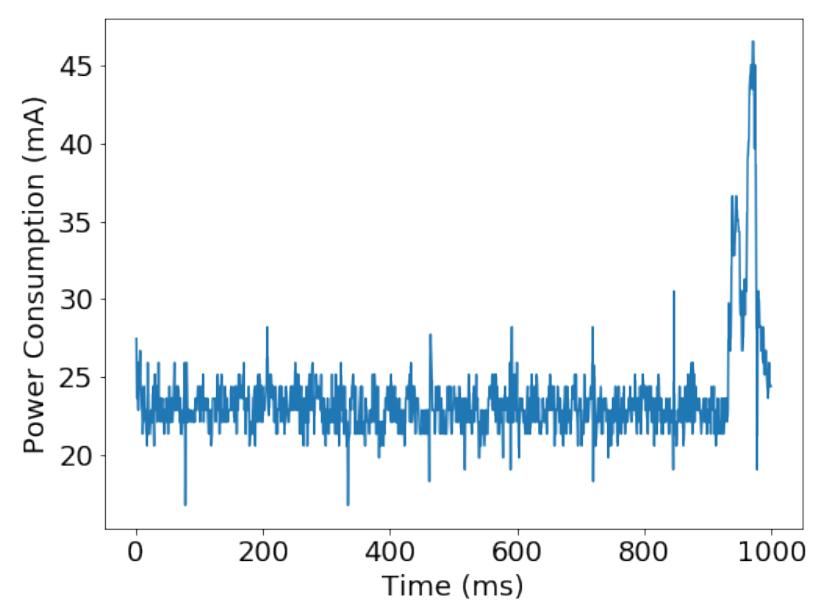

Fig. 5: Mesh consumption transmission peak.

Fig. 5 shows a chart of power consumption by time, in milliseconds. We can see that there is a peak of energy consumption when the module sends the data. The transmission time is around $35 \mathrm{~ms}$, which represents $3.5 \%$ of 1 second.

$$
B L=\left(\frac{B C}{C R}\right) \times(1-D S)
$$

To calculate the battery lifetime, we consider that the module is transmitting $3.5 \%$ of the time and $96.5 \%$ in idle mode because, in the agricultural scenario, there is no necessity to update the parameters for a short period. Also, we use a reference battery with a capacity of 9,800 $\mathrm{mAh}$, and the estimated battery lifetime was 262.65 hours.

Although the mesh protocol has a higher consumption, it still has a low cost of energy consumption. Though it is possible using photovoltaic solar panels to charge the sensor nodes battery, and even on cloudy days, the battery can keep the node running for approximately 11 consecutive days.

\section{Topology Comparison}

We have performed a comparison of two WSN with different Bluetooth topologies, the piconet and mesh. In our analysis, we considered the results of the communication distance test of Section $\mathrm{V}$. We noticed that in the network using the piconet Bluetooth topology Fig. 6a, sensor nodes (slaves) 
located in the corners of the field were out of the communication range of the master node. For all nodes to be part of the network, we should include other master nodes $6 \mathrm{~b}$ in a Scatternet scheme. Moreover, this makes node placement more complicated, as it is necessary to check where a master node should be.

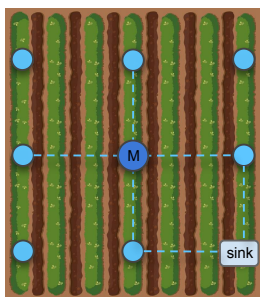

(a) Piconet

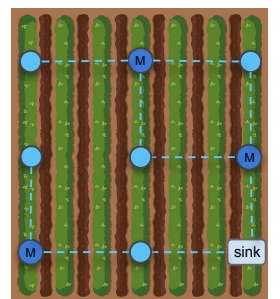

(b) Scatternet

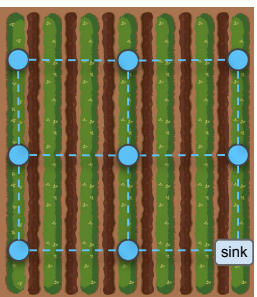

(c) Mesh
Fig. 6: Bluetooth Topology Comparison.

The BLE mesh topology uses a flat model whereby all nodes have the same hierarchical level. Consequently, it does not present the problem of the topology mentioned above since every node can connect to any other nodes nearby, as shown in Fig. 6c. Another benefit of the mesh network when compared to the Scatternet, is in the absence of a single point of failure, since there is no single access point to the network.

\section{CONCLUSIONS AND Future WORK}

This paper presented the design of a system for monitoring agricultural crops. Applications for this scenario have as main requirements the scalability, coverage, and also long battery life, due to the large dimensions of crop fields. Our Bluetooth mesh sensor network shown to be suitable for the proposed scenario due to good coverage and easy scalability allowed by the mesh topology, and to the low power consumption of the Bluetooth Low Energy. Furthermore, the comparison of the Bluetooth network topologies presented the advantages of using mesh topology in a WSN.

Future work involves a comparison between the flooding mechanism of our mesh sensor network and a mesh network that uses a routing protocol in order to evaluate the energy consumption and the fitness of both mechanisms in the purposed application scenario.

\section{REFERENCES}

[1] UN (2017). "World Population Prospects The 2017 Revision", 2017. [Online]. Available: https://population.un.org/ wpp/Publications/Files/WPP2017_Volume-II-DemographicProfiles.pdf

[2] Statista. Cotton production value in the U.S. from 2000 to 2018 (in 1,000 U.S. dollars). [Online]. Available: https://www.statista.com/statistics/191505/cotton-productionvalue-in-the-us-since-2000/

[3] Statista. Top 10 U.S. states based on production value of corn for grain in 2017 (in 1,000 U.S. dollars). [Online]. Available: https://www.statista.com/statistics/191052/top-usstates-by-production-value-of-corn-for-grain/

[4] R. Radha, A.K. Tyagi, K. Kathiravan, G. Staflin Betzy (2020) Precision Crop Protection Using Wireless Sensor Network. In: Abraham A., Cherukuri A., Melin P., Gandhi N. (eds) Intelligent Systems Design and Applications. ISDA 2018 2018. Advances in Intelligent Systems and Computing, vol 940. Springer, Cham

[5] K. Nickelsen, Explaining Photosynthesis: Model of Biochemical Mechanisms 1840-1960. Dordrecht, Netherlands: Springer, 2015, pp. 94-95.

[6] C. Gull, M. T. Minkov, E. G. Pereira and J. A. M. Nacif, A Low-Cost Chlorophyll Fluorescence Sensor System, IEEE Conf. on Brazilian Symposium on Computing Systems Engineering (SBESC), pp. 186 - 191.

[7] T. Rault, A. Bouabdallah, Y. Challal, Energy efficiency in wireless sensor networks: A top-down survey, Computer Networks, Volume 67, 2014, pp. 104-122.

[8] M. S. Mekala and P. Viswanathan, "A Survey: Smart agriculture IoT with cloud computing,"2017 International conference on Microelectronic Devices, Circuits and Systems (ICMDCS), Vellore, 2017, pp. 1-7.

[9] X. Shi, X. An, Q. Zhao, H. Liu, L. Xia, X. Sun, and Y. Guo, "State-of-the-Art Internet of Things in Protected Agriculture," Sensors, vol. 19, no. 8, p. 1833, Apr. 2019

[10] P. Zenker, S. Krug, M. Binhack, J, Seitz (2016). Evaluation of BLE Mesh capabilities: A case study based on CSRMesh. 2016 Eighth International Conference on Ubiquitous and Future Networks (ICUFN),pp. 790-795.

[11] K. Nickelsen, Explaining Photosynthesis: Model of Biochemical Mechanisms 1840-1960. Dordrecht, Netherlands: Springer, 2015, pp. 94-95.

[12] Qualcomm Bluetooth Low Energy Solutions, CSRmesh and CSR1010, Qualcomm Technologies International.

[13] Bluetooth Core Specification Version 4.1, Bluetooth SIG.

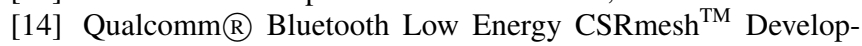
ment Kit.

[15] MQTT.org, What is MQTT? [Online]. Available: http://mqtt.org/faq

[16] V. Karagiannis, P. Chatzimisios, F. Vazquez-Gallego, J. Alonso-Zarate (2015), A Survey on Application Layer Protocols for the Internet of Things, Transaction on IoT and Cloud Computing 3 (1), pp. 11-17 